\title{
2. MENORES REINCIDENTES Y NO REINCIDENTES EN EL SISTEMA DE JUSTICIA JUVENIL ANDALUZ*
}

\section{Minors: repeat offenders and non-repeat offenders in the juvenile justice system in Andalusia}

\author{
Elisa García-EsPañA** \\ Octavio García PÉREZ*** \\ María José Benítez JimÉNEZ**** \\ FÁtIMA PÉREZ JIMÉNEZ *****
}

\section{Resumen}

En los últimos años han visto la luz varios informes sobre la reincidencia juvenil en el ámbito de distintas comunidades autónomas. Dentro del marco de un proyecto más amplio sobre seguridad ciudadana, se diseñó una investigación con la pretensión de

\footnotetext{
* $\quad$ Recibido: $12 / 11 / 2010$

Revisado: 01/12/2010

Aceptado: 07/02/2011

Institución responsable que soporta la investigación y/o soporte financiero (en su caso): Programa Nacional de Investigación Científica, Desarrollo e Innovación Tecnológica de la Secretaría de Estado de Universidades e Investigación del Ministerio de Educación y Ciencia (SEJ2006-07242/JURI).

** Elisa García-España

Responsable de correspondencia.

Instituto andaluz de Criminología de la Universidad de Málaga. Universidad de Málaga. Campus de Teatinos s/n. Edificio de Investigación, $1 .^{a}$ planta.

29730 Málaga. España

Tel: $952132323 / 33$

E-mail: elisa@uma.es

*** Octavio García Pérez

Instituto andaluz de Criminología de la Universidad de Málaga. Universidad de Málaga.

**** María José Benítez Jiménez

Instituto andaluz de Criminología de la Universidad de Málaga. Universidad de Málaga.

****** Fátima Pérez Jiménez

Instituto andaluz de Criminología de la Universidad de Málaga. Universidad de Málaga.
} 
abordar las variables legales y/o sociales que distinguen a los reincidentes de los no reincidentes en Andalucía.

Con este trabajo, partiendo del marco legal constituido por la Ley Orgánica 5/2000, de 12 de enero, y sus sucesivas reformas, presentamos la reiteración delictiva de los menores obtenida a partir de una muestra de expedientes judiciales del sistema de justicia penal juvenil, así como una descripción panorámica de las variables personales, sociales y legales que nos permiten diferenciar con un análisis bivariable y de forma estadísticamente significativa, al grupo de menores reincidentes del de los no reincidentes en los tres juzgados andaluces analizados.

Palabras clave: menores, reincidencia, delincuencia juvenil, sistema de justicia juvenil.

\begin{abstract}
During the last few years many reports about juvenile repeat offenders have come to light in different Autonomous Communities. Within the framework of a larger project that deals with public security, this research project was designed in order to research the legal and/or social variables that distinguish repeat offenders from non repeat offenders in Andalusia.

In this work, which takes into account the legal framework provided by the Organic Law 5/2000 from 12 January and its subsequent amendments, we present the repetition of criminal behavior of minors based on a sample of court files from the juvenile criminal justice system. Moreover, a panoramic description of the personal, social and legal variables are presented that enable us to distinguish, using bivariate analysis and in a statistically significant manner, the repeat offender group of minors from the non repeat offenders in the three Andalusian courts used in this study.
\end{abstract}

Keywords: minors, repeat offenders, juvenile delinquency, juvenile justice system.

\title{
I. Introducción
}

En nuestro país, en los últimos años se ha despertado un gran interés por el análisis de diversos aspectos relacionados con la reincidencia de menores, incluyéndose en todos un cálculo de la tasa de reincidencia. Así, según un estudio realizado en Cataluña, el nivel de reincidencia dentro del sistema penal juvenil de esta comunidad es de un 22.7\% (Capdevilla, 2005). En esta investigación la muestra estaba integrada por todos los menores que hubiesen finalizado la medida judicial impuesta en 2002 , siguiéndose su trayectoria durante 2 o 3 años para comprobar si habían reincidido, entendiendo que lo habían hecho si tenían abiertas causas posteriores en la fiscalía de menores. En otro estudio, realizado en el País Vasco, se calculó la tasa de reincidencia de la muestra total en un 28.1\% (San Juan y Ocaríz, 2009). Este trabajo también usa como criterio de selección muestral la finalización de la medida judicial impuesta y aprecian 
la reincidencia cuando aparecen nuevas infracciones en el sistema de justicia penal juvenil y tras haber alcanzado la mayoría de edad, siendo el periodo de seguimiento entre 2003-2007. En Asturias se ha llevado a cabo otro en el que se constata que el porcentaje de reincidentes asciende al 30\% (Bravo; Sierra; Del Valle, 2007). En este supuesto se amplía el criterio de selección al incluirse a todos los menores que hubieran cumplido una medida judicial entre enero de 2001 y diciembre de 2004. La reiteración delictiva se constató a partir de los hechos posteriores que constaran en los expedientes y el periodo de seguimiento alcanzó hasta un máximo de 4 años. Por último, en la investigación efectuada en la Comunidad autónoma de Madrid, el 26.9\% de la muestra era reincidente (Graña et al., 2008). La definición de la reincidencia fue retrospectiva, sin embargo la selección muestral es diferente a los otros estudios, atendiendo a la especificidad de su objetivo. En Madrid, la muestra estuvo conformada por todos los menores que ingresaban en centros de internamiento de dicha comunidad durante todo un año, y a los que se les administraba a su ingreso tres instrumentos de predicción de la reincidencia con el objetivo de comprobar su utilidad en el contexto del diseño y ejecución de los programas de intervención.

A pesar del número de estudios que abordan la tasa de reincidencia en las distintas comunidades autónomas de España, hay que tener en cuenta que dichas tasas pueden ser dispares atendiendo a la diferente metodología llevada a cabo para su cálculo. De hecho, en los estudios anteriores encontramos diferencias, al menos en la selección muestral, el periodo de seguimiento y la definición de la reincidencia. Con respecto a otros estudios internacionales, además, otras dificultades a sortear serían las diferentes franjas de edad sobre las que se calcula dicha reincidencia.

En nuestra investigación, los datos se obtienen de los expedientes que obran en tres juzgados del sistema de justicia de menores en Andalucía y la reiteración delictiva se analiza tras un seguimiento de, al menos, dos años.

\section{Marco legal}

\subsection{Catálogo de sanciones}

El catálogo de sanciones de la Ley reguladora de la responsabilidad penal de los menores (Ley Orgánica 5/2000, en adelante LORRPM) durante el periodo de tiempo objeto de esta investigación, no difiere sustancialmente del vigente. La diferencia radica en que en la actualidad se contempla en el apartado i) del art. 7.1 la medida de "prohibición de aproximarse o comunicarse con la víctima o con aquellos de sus familiares u otras personas que determine el Juez». En 
realidad, con la antigua regulación existían mecanismos para aplicarla a los menores como, por ejemplo, acudiendo a una libertad vigilada en la que se impusiera como regla de conducta la prohibición de aproximarse o comunicarse con la víctima u otras personas (Gutiérrez, 2007). Asimismo, tras la reforma realizada por la Ley Orgánica 8/2006, se indica expresamente que el internamiento terapéutico se puede cumplir en régimen abierto, semiabierto y cerrado, pero, a diferencia de lo que sucede con el internamiento normal en el que cada uno de los regímenes se recogen en apartados diferentes, en el terapéutico se alude a los tres en el mismo apartado.

La LRRPM, en la versión vigente al tiempo en el que se hace la investigación, contiene un amplio catálogo de sanciones. Aunque es posible utilizar diversos criterios de clasificación, a nuestro entender, el más relevante es aquel que atiende a la naturaleza privativa de libertad o ambulatoria. Y ello porque una directriz fundamental del Derecho penal de menores es el principio de subsidiariedad. El art. 7.1 contempla cinco sanciones privativas de libertad: internamiento en régimen cerrado, internamiento en régimen semiabierto, internamiento en régimen abierto, internamiento terapéutico y permanencia de fin de semana. Las demás sanciones que recoge son no privativas de libertad: tratamiento ambulatorio, asistencia a un centro de día, libertad vigilada, convivencia con otra persona, familia o grupo educativo, prestaciones en beneficio de la comunidad, realización de tareas socio-educativas, amonestación, privación del permiso de conducir para ciclomotores o vehículos a motor, o del derecho a obtenerlo, o de las licencias administrativas para caza o para uso de cualquier tipo de armas, e inhabilitación absoluta.

El art. 7.1 parece dar a entender que las sanciones se recogen en él clasificadas en función de su gravedad, pues alude a medidas «ordenadas según la restricción de derechos que suponen»(Ornosa, 2003; Aguirre, 2000). Esta idea ya planteaba problemas con la redacción original de este precepto, puesto que la privación del derecho de conducir o de licencia de caza o armas es más grave que la amonestación (Aguirre, 2000) y, en nuestra opinión, la permanencia de fin de semana o la libertad vigilada no son, en general, menos graves que el tratamiento ambulatorio o la asistencia a centro de día. Con la reforma operada por la Ley Orgánica 7/2000, de 22 de diciembre, en la que se introdujo la inhabilitación absoluta como última medida, cabe afirmar que aunque el art. 7.1 tuviera la pretensión de ordenar las medidas en atención a su gravedad, lo cierto es que no lo ha conseguido, debiéndose interpretar como una mera enumeración carente de cualquier criterio clasificatorio. 


\subsection{Duración de las medidas}

La cuantía de las diferentes sanciones depende de la franja de edad a la que se aplique. La regla general la encontramos en el art. 9. 3.a: la duración máxima de las medidas es de dos años salvo la de prestaciones en beneficio de la comunidad, que no podrá superar las cien horas, y la de permanencia de fin de semana que no podrá exceder de los ocho fines de semana.

No obstante, esta regla general tiene varias excepciones. Así, para los mayores de 16 años, la regla $4{ }^{\text {a }}$ del art. 9 permite que las sanciones duren hasta 5 años siempre que el delito haya sido cometido con violencia o intimidación en las personas o con grave riesgo para la vida o la integridad física de las mismas y, además, el equipo técnico lo recomiende en su informe. En estos casos, las prestaciones en beneficio de la comunidad pueden alcanzar las 200 horas y, la permanencia de fin de semana, los 16 fines de semana. En los casos de extrema gravedad del art. 9.4. ${ }^{a}$ en el apartado cinco, dispone que se impondrá una medida de internamiento en régimen cerrado de uno a 5 años más una libertad vigilada de hasta 5 años, es decir, aquí la medida puede llegar a durar hasta 10 años.

Por último, la disposición adicional cuarta que fue introducida por la Ley Orgánica 7/2000, de 22 de diciembre, estableció que para los delitos muy graves (homicidio, asesinato, violación, agresiones agravadas del art. 180, delitos de terrorismo y delitos castigados con penas de prisión de 15 o más años) se impondrán medidas de internamiento en régimen cerrado de hasta 4 o 5 años más una libertad vigilada de hasta 3 años para los menores de hasta 16 años y de 8 o 10 años de internamiento en régimen cerrado más una libertad vigilada de hasta 5 años para los de hasta 18 años. De este modo, para los casos más graves, la sanción puede durar hasta 15 años.

\subsection{Los presupuestos para la aplicación de las sanciones}

Requisito imprescindible para exigir responsabilidad penal a un menor es que haya cometido un hecho que esté tipificado como delito o falta en el Código Penal o en la legislación penal especial (art. 1.1). Esto significa que, para los menores, los hechos constitutivos de delito son los mismos que para los adultos, es decir, el Derecho penal de menores es accesorio del de estos en lo relativo a las infracciones. Hay que destacar que no basta con la realización de un hecho típico sino que, como se encarga de aclarar el art. 5.1, es preciso que «no concurra en ellos ninguna de las causas de exención o extinción de la responsabilidad criminal previstas en el vigente Código Penal». No obstante, si concurriera alguna causa de inimputabilidad, el art. 5.2 dispone que se podrán aplicar las medidas de internamiento terapéutico o tratamiento ambulatorio. 
En segundo lugar, es preciso que el infractor se encuentre comprendido dentro de la franja de edad a la que es aplicable la LORRPM. En concreto, es necesario que los menores de edad responsables del delito tengan más de 14 años y menos de 18. Aunque en principio, en virtud de lo dispuesto en el art. 69 del Código, la LORRPM era aplicable también a los jóvenes de 18 a 21 años en los términos contemplados en el art. 4, lo cierto es que durante el periodo de tiempo objeto de la investigación esta regulación no estaba vigente, pues la entrada en vigor del art. 4 había sido suspendida.

En tercer lugar, aunque la LORRPM no lo diga expresamente salvo en el caso del internamiento (Boldova, 2002; Sánchez, 2000), si las sanciones se orientan a la prevención especial, estas se tienen que asentar sobre un pronóstico de peligrosidad criminal (Feijoo, 2001; Vargas, 2001). De ahí que el texto legal haga referencia a los factores que permiten realizarlo. Así el art. 7.3 establece que para la elección de la medida adecuada se atienda especialmente, además de a los hechos realizados, «a la edad, las circunstancias familiares y sociales, la personalidad y el interés del menor»... Por ello, lo relevante para imponer una sanción no puede ser la mera existencia de necesidades educativas. No es función del Derecho penal cubrir cualquier déficit educativo, circunstancia que en la franja de edades de las que se ocupa la Ley se da en todos, puesto que aún no se han completado los procesos de socialización, sino únicamente aquellos extraordinarios que puedan conducir a reiterar la comisión de hechos delictivos.

Por último, es necesario que esa peligrosidad no pueda paliarse a través de las agencias de socialización informales (familia, escuela, etc.) y que tampoco pueda serlo por medio de una mediación. Si fuera el caso, procedería aplicar el art. 18 o el 19 LORRPM.

\subsection{Criterios para la elección de la sanción y determinación de su magnitud}

A diferencia de lo que acontece en el Código Penal, en la LORRPM no se establece una vinculación entre cada delito y la sanción que le corresponde. El planteamiento del art. 7.3 es coherente con la finalidad preventivo-especial que preside la regulación. Si de lo que se trata es de eliminar aquellos déficit educativos que llevan al menor a cometer los delitos, la sanción adecuada no se puede conocer de antemano, sino que dependerá de los factores personales, familiares y sociales que concurran en cada sujeto, es decir, el hecho cometido no es más que un factor más a valorar de cara a identificar las necesidades que presenta el menor y cuál es la medida idónea para hacerle frente. 
Ahora bien, discrecionalidad no significa arbitrariedad. En efecto, la decisión sobre la sanción y su magnitud ha de respetar unas pautas que la propia Ley ha establecido explícitamente y otras que no se mencionan, pero que son una consecuencia inmediata de los fines que se persiguen.

En primer lugar, la LORRPM parece adoptar el principio de la medida única, salvo las excepciones contempladas en la misma (Aguirre, 2000), si bien recientemente se ha incorporado el principio de acumulación de penas con algunas limitaciones (críticamente García, 2008). Así, en primer lugar, la acumulación de sanciones se preveía en el caso del concurso real, a diferencia de lo que acontecía en el concurso ideal y el delito continuado, solo cabía imponer una única sanción, tomando como punto de referencia la más grave. A lo anterior había que añadir lo previsto para las medidas terapéuticas en las que se mencionaba que podían imponerse conjuntamente con otras o la privación del derecho de conducir o del uso de armas que podían aplicarse como accesorias.

A partir de aquí, para elegir la medida y fijar su duración, el Juez de Menores debe respetar el principio de proporcionalidad. Así, el art. 8.2 dispone que la duración de las medidas privativas de libertad no podrá exceder «del tiempo que hubiera durado la pena privativa de libertad que se le hubiere impuesto por el mismo hecho, si el sujeto, de haber sido mayor de edad, hubiera sido declarado responsable, de acuerdo con el Código Penal». Aun cuando solo se aplica a las medidas privativas de libertad, este límite rige también para las no privativas de libertad (Aguirre, 2000; STC 36/1991, de 14 de febrero).

También el art. 9 contenía varias reglas reconducibles a este principio. En primer lugar, los hechos de escasa gravedad, las faltas, solo se podrán castigar con amonestación, permanencia de fin de semana hasta un máximo de cuatro fines de semana, prestaciones en beneficio de la comunidad hasta cincuenta horas, y privación del permiso de conducir o de otras licencias administrativas. La medida de internamiento en régimen cerrado solo se puede aplicar en delitos en cuya comisión se haya empleado violencia o intimidación en las personas o actuado con grave riesgo para su vida o integridad física. Además, los hechos cometidos por imprudencia, por muy graves que sean, nunca pueden ser sancionados con el internamiento en régimen cerrado. Estos límites suelen vincularse por la doctrina al principio de proporcionalidad (Tamarit, 2001; Sánchez, 2000; Gómez, 2001; González y Cuerda, 2002).

Para Gómez (2001:173) «se echa en falta una previsión que recortase la posibilidad de imponer sanciones graves a otros supuestos que, si bien determinan la aplicación de la LORRPM conforme a las previsiones de su art. 1, merecen igualmente un menor contenido de desvalor; en concreto, las formas de partici- 
pación intentadas, los actos de participación así como los actos preparatorios punibles». A lo anterior añade que hubiera sido conveniente «que la Ley hubiera recogido de forma expresa un principio que consagra el art. 95.2 del Código Penal a propósito de los adultos: que cuando la pena que hubiera correspondido al delito cometido no fuera privativa de libertad, no podrá imponerse una medida que prive de libertad al menor». Con relación a la primera cuestión, aun siendo cierto -y de hecho en algún proyecto se hizo referencia a algunos de estos aspectos-, al consagrarse el principio de proporcionalidad, como se desprende de los arts. 8 y 9 , hasta cierto punto el problema se puede resolver por vía interpretativa. En este sentido, señala Colás (2002:66) que los grados de ejecución se han de tener en cuenta de cara a establecer cuál es la duración máxima de la pena privativa de libertad que le hubiere podido corresponder de ser adulto para aplicar el límite del art. 8.2. En cuanto al segundo problema suscitado por la autora, si los internamientos no pueden durar más de lo que hubiere durado la pena privativa de libertad que le habría correspondido de ser adulto, si el hecho no está castigado con esta, no cabrá aplicarle dicha sanción.

En los casos de la regla $4 .{ }^{a}$ del art. 9 donde se apreciara extrema gravedad es obligatorio imponer una medida de internamiento en régimen cerrado de 1 a 5 años a la que seguiría una de libertad vigilada de hasta 5 años. El propio precepto aclaraba que la reincidencia tendría la consideración de supuesto de extrema gravedad. La regulación de las hipótesis de extrema gravedad se completaba con lo que estipulaba la disposición adicional cuarta.

En el caso de menores en los que concurra alguna causa de inimputabilidad, únicamente se podrán imponer las medidas de internamiento terapéutico o tratamiento ambulatorio.

\section{Material y método}

\subsection{Objetivos y selección muestral}

Esta investigación persigue, como objetivo general, abordar la reincidencia de los jóvenes que pasan por el sistema de justicia juvenil andaluz. De forma específica, se busca conocer la tasa de reincidencia de los jóvenes andaluces, los factores que permiten establecer diferencias entre los jóvenes reincidentes y no reincidentes, y los predictores de la reincidencia en un área geográfica determinada como es Andalucía.

La población objeto de nuestro estudio se compone de todos los jóvenes sobre los que recayó una sentencia judicial en el ámbito del sistema de justicia juvenil en el año 2002 en Andalucía. 
Nuestra muestra la escogimos de tres juzgados de diferentes provincias, concretamente de Granada, Málaga y Sevilla. En dichos juzgados se extrajo de forma aleatoria una muestra de las 1.594 sentencias existentes en los libros de sentencias de 2002, siguiendo el orden cronológico en el que estaban anotadas las resoluciones.

Tras la selección aleatoria de las sentencias, se procedió a la búsqueda del expediente judicial del menor o menores implicados para recabar información sobre asuntos anteriores y posteriores a la causa base por la que fue sentenciado en 2002. Con este método se recopiló información de 590 menores, de los que pudimos obtener una imagen de su carrera delictiva, así como de las respuestas que les otorga el sistema de justicia juvenil .

\subsection{Recogida de datos e instrumento}

Para la recogida de datos, en los expedientes judiciales de menores se elaboró una ficha técnica que finalmente estaba compuesta de 79 variables personales, sociodemográficas, legales y procedimentales. Para su validación se procedió a un proyecto piloto con 20 casos en un juzgado de Málaga. Con dicho estudio previo se comprobó que en los expedientes no se recogía información sobre todas las variables que eran de nuestro interés. De ahí que se eliminaran de nuestra propuesta originaria aquellas variables sobre las que no se podía obtener datos.

Finalmente, la ficha técnica quedó dividida en cinco apartados, los cuales tienen un orden determinado para facilitar a los becarios del proyecto la recolección de los datos. El primero agrupa la información relativa a la identificación del menor y de la causa base; el segundo recolecta datos de la infracción y del procedimiento judicial de la causa base y de todas las causas anteriores y posteriores a aquella hasta un máximo de tres; los tercer, cuarto y quinto son los destinados a reflejar los datos personales, familiares y del entorno social del menor respectivamente. En estos tres apartados los datos se recogieron solo de la causa base.

A raíz de dicha ficha, se diseñó una base de datos en el programa SPSS 15.0 sobre la que se volcaron los datos directamente desde los expedientes, eliminando los riesgos que se corren con pasos intermedios tales como extraerlos mediante una ficha en papel para después proceder a su informatización. Otra ventaja de este procedimiento es que se ahorra tiempo. Pese a todo, lo cierto es que la obtención de todos los datos llevó más de 18 meses, ya que para recoger la información requerida de las causas anteriores y posteriores a la causa base había que hacer una meticulosa labor de búsqueda de los expedientes, incluso acudir, en algunas ocasiones, a otros juzgados. La ausencia de una base de datos 
judicial centralizada donde se recopilen todas las informaciones que necesitábamos hace que trabajos empíricos de esta envergadura sean costosos y tediosos, además de necesitarse controles sobre el trabajo de los becarios para evitar errores en la recolección de datos.

\subsection{Procedimiento para llevar a cabo el trabajo de campo}

Para acceder a los datos de los expedientes judiciales se elevó una solicitud al Tribunal Superior de Justicia de Andalucía basada en que el trabajo de investigación empírico que pretendíamos realizar entraba dentro del concepto de publicidad de los libros, resoluciones y archivos judiciales que para «cualquier interesado» establece el art. 266.1 de la LOPJ en relación con el art. 235 de la misma Ley. Así lo ha reconocido también el Pleno del Consejo General del Poder Judicial en acuerdo de 15 de noviembre de 1989 (Documento 152, publicado en febrero de 1990). Además de hacer constar el marcado interés público y general de esta investigación, nos comprometimos a que todos los datos personales contenidos en dichos expedientes serían tratados con el máximo celo y reserva, y que en el análisis y exposición de los datos en ningún caso se vulneraría el derecho a la intimidad de los afectados. El Tribunal Superior de Justicia nos remitió directamente a cada uno de los titulares de los juzgados de menores en los que queríamos hacer el estudio. Los juzgados de Málaga y Granada accedieron gratamente a nuestra solicitud. Sin embargo, uno de los juzgados de Sevilla no autorizó que un trabajo de campo de esas características se llevara a cabo en su juzgado. De ahí que remitiéramos la solicitud a un segundo juzgado, cuyo titular se mostró desde el primer momento dispuesto a colaborar en lo que fuera necesario.

\section{Resultados: reincidencia según expedientes judiciales}

La causa base es el punto de inflexión de nuestra investigación. Entendemos por reincidencia para este estudio la reiteración de hechos delictivos tras la causa base y antes de alcanzar la mayoría de edad. Los antecedentes que constan en el expediente judicial anteriores a nuestro punto de partida nos muestran las veces que el sistema ha actuado sobre el menor.

\subsection{Tasa de reincidencia}

En nuestro estudio, de los 590 jóvenes que recibieron una sentencia por los juzgados de menores estudiados, $163(27.6 \%)$ reiteraron un comportamiento delictivo antes de alcanzar la mayoría de edad. 
De los 163 jóvenes con reincidencia judicial, la mayoría (40.5\%) era de Málaga, y esta provincia es la que tiene un porcentaje de jóvenes reincidentes (33.8\%) mayor que el resto de las provincias (27.7\% Sevilla y $21.5 \%$ Granada).

La mayoría de jóvenes $(57.4 \%, \mathrm{n}=339)$ no tiene antecedentes ni reincidencia judicial en el ámbito de menores. Entre los 159 jóvenes que tenían causas anteriores a la causa base, el 55.3\% no volvió a infringir la Ley. Entre el grupo que había reincidido antes de alcanzar la mayoría de edad, el 56.4\% no tenía antecedentes en el juzgado de menores y, por lo tanto, la causa base era su primera causa judicial. Solo el $12 \%$ de los jóvenes contaba con causas anteriores y posteriores a la causa base $(n=71)$. Véase la siguiente tabla $n .^{\circ} 1$ explicativa de lo que aquí se expone.

Tabla 1: Causas anteriores y posteriores a la causa base en el ámbito de la justicia juvenil

\begin{tabular}{|l|l|l|l|}
\hline & \multicolumn{2}{|l|}{ Reincidencia } & Total \\
\hline Antecedentes & SÍ & NO & \\
\hline SÍ & 71 & 88 & 159 \\
$\%$ de antecedentes & $44,7 \%$ & $55,3 \%$ & $100 \%$ \\
$\%$ con la reincidencia & $43,6 \%$ & $20,6 \%$ & $26,9 \%$ \\
\hline NO & 92 & 339 & 431 \\
$\%$ de antecedentes & $21,3 \%$ & $78,7 \%$ & $100 \%$ \\
$\%$ con la reincidencia & $56,4 \%$ & $79,4 \%$ & $73,1 \%$ \\
\hline Total & 163 & 427 & 590 \\
$\%$ de antecedentes & $27,6 \%$ & $72,4 \%$ & $100 \%$ \\
$\%$ con la reincidencia & $100 \%$ & $100 \%$ & $100 \%$ \\
\hline \multicolumn{2}{|c|}{$\left(x^{2}=31,560^{a} ; \mathrm{gl}=1 ; \mathrm{sig}=, 000\right)$} \\
\hline
\end{tabular}

De la tabla anterior es posible deducir dos grupos de menores: uno de ellos estaría formado por los menores que puntualmente pasan por el sistema de justicia juvenil, ya que no tienen antecedentes ni reincidencia, siendo este el grupo más numeroso (57.4\%); y otro, compuesto por los menores que han cometido más de una infracción, antes, después o antes y después de la causa base, y en el que se agrupa el $42.5 \%$ de la muestra.

\subsection{Diferencias entre menores reincidentes y no reincidentes}

En este apartado presentamos las diferencias más sobresalientes entre el grupo de menores reincidentes $(n=163)$ y el de no reincidentes $(n=427)$. 
Entre los reincidentes, el 96.3\% son chicos. Aunque el resultado parece corroborar el hecho ampliamente demostrado de que los hombres están más representados en las conductas infractoras que las mujeres, estas diferencias estadísticas, en este caso, no son significativas $\left(x 2=2.571^{a}\right.$; $g l=1$; $\left.s i g=.109\right)$.

La mayoría de los menores de la muestra que reincidieron tenían 16 años en el momento de la comisión de la causa base (35\%). Centrándonos en la edad de la primera infracción, aquellos que cometieron su primer hecho con 13 y 14 años son los que presentan mayor porcentaje de reincidencia (37.5\% en ambos grupos de edad), disminuyendo la reincidencia conforme aumenta la edad. De hecho, en el grupo de menores que realizaron su primera infracción con 17 años, solo un $14.7 \%$ reincidieron $\left(x 2=28.283^{a} ; g l=5\right.$; $\left.s i g=.000\right)$. Este resultado tiene un valor muy limitado, ya que poco puede decirnos la jurisdicción de menores sobre la reincidencia de los menores de 17 años.

El porcentaje de jóvenes extranjeros que reinciden (25.7\%) es ligeramente inferior al de españoles (27.7\%). En el grupo de reincidentes, y debido a que los extranjeros tienen escasa representatividad entre la población objeto de estudio -tan solo el 5.9\% de la muestra no son de origen español-, el porcentaje de extranjeros es el 5.5\%. Estas diferencias son significativas, por lo que se puede afirmar que dicho resultado no se debe simplemente a la elección aleatoria de la muestra $\left(x 2=.068^{a} ; g l=2 ; \operatorname{sig}=.020\right)$.

Reinciden más los jóvenes que no trabajan (31.5\%) que los que lo hacen (20.8\%). En el grupo de reincidentes, el 27.6\% tenía una ocupación laboral frente al $64.3 \%$ que no la tenía. En esta ocasión, las diferencias sí son significativas $\left(x 2=7.866^{a} ; g l=1 ; \operatorname{sig}=.794\right)$.

El absentismo escolar no parece diferenciar entre los menores reincidentes y no reincidentes, ya que el $42.3 \%$ de los primeros y el $45.2 \%$ de los segundos nunca acuden a clase. Sin embargo, entre los reincidentes hay un mayor número de menores que acuden irregularmente a clase (15.3\%) que de no reincidentes $(6.1 \%)$, siendo las diferencias significativas $\left(x 2=14.865^{a} ; g l=3\right.$; sig $=$ .002). Este dato es relevante, teniendo en cuenta que entre los que nunca asisten a clase hay menores que trabajan (47.3\%). Por ello el absentismo escolar no es sinónimo de ociosidad. En nuestra muestra, el grupo de jóvenes que ni estudia ni trabaja asciende al $24.4 \%$, de los cuales el $45.1 \%$ reincidieron. Hay que destacar también que entre los menores de nuestra muestra hay un 5\% que estudia y trabaja, de estos solo un $20 \%$ reincidió en su comportamiento delictivo.

También el retraso escolar es una de las variables que la doctrina relaciona con las carreras delictivas. Entre los menores andaluces, el 49.1\% de los reincidentes sufrían un retraso escolar $\left(x 2=15.194^{a} ; g l=2 ; \operatorname{sig}=.001\right)$. Entre los no reincidentes, el índice de retraso escolar es inferior (31.9\%). 
De los reincidentes, el 28.2\% manifestó una conducta problemática dentro del aula frente al $35 \%$ que no tuvo ese tipo de comportamiento. Entre los no reincidentes, las diferencias entre los que tuvieron o no un comportamiento problemático en el aula son mayores, ya que solo el $11.9 \%$ tuvo ese tipo de comportamiento frente a un $45 \%$ que no se comportó de forma problemática $\left(x 2=22.929^{a} ; g l=2 ; s i g=.000\right)$.

En muchos casos no encontramos información sobre el consumo de drogas del menor y el cruce de esta variable con la dependiente «reincidencia» no presenta diferencias estadísticamente significativas.

Existe poca diferencia entre los menores reincidentes y no reincidentes cuando analizamos si son consumidores de alcohol, y estas diferencias tienen una significancia estadística del .052. Así, el 31.3\% de los que consumían alcohol reincidieron, frente a un $29.3 \%$ que no lo hacía. Si nos centramos en el grupo de reincidentes, la mayoría de ellos, con un $68.7 \%$ de los casos, no consumía alcohol $\left(x 2=5.913^{a} ; g l=2 ; s i g=.52\right)$.

Entre los que reinciden, la mayoría tiene anotado en su expediente que se comporta de modo problemático (57.1\%). La diferencia con los no reincidentes es que estos tienen mayoritariamente (63\%) un comportamiento normalizado. Además, entre el grupo de estos últimos, el 79.8\% no reincide, mientras que entre los menores con comportamiento problemático la representatividad de los no reincidentes desciende hasta un 54.6\% ( $x 2=54.068^{a} ; \mathrm{g} l=2$; sig $\left.=.000\right)$.

El número de menores que presentan problemas de salud mental es muy escaso $(n=21)$. Pero, centrándonos en las diferencias entre el grupo de reincidentes y no reincidentes, hay que destacar que entre los primeros hay un número algo superior de menores con problemas de salud mental (5.5\%) que entre los no reincidentes (2.8\%). Por otra parte, los menores con problemas de salud mental tienen unos porcentajes de reincidencia (42.9\%) superior al de los menores que no presentan problemas de salud mental (28.4\%), siendo las diferencias estadísticamente significativas $\left(x 2=6.201^{a}\right.$; $g l=2$; $\left.s i g=.045\right)$.

Los menores con problemas de salud física son aún menos $(n=8)$, pero en la distinción entre los dos grupos -reincidentes y no reincidentes- se observa una ligera mayor representación de menores con problemas de salud entre los reincidentes $(1.8 \%)$ que en los no reincidentes $(1.2 \%)\left(x 2=4.316^{a} ; g l=2 ;\right.$ sig $\left.=.116\right)$.

Con relación a la variable «maltrato en el entorno familiar», el número de casos en los que se produjo también es reducido ( $n=27$ agrupando el maltrato sufrido directa e indirectamente). Solo no consta el dato en 68 casos. La cuestión es si esta experiencia en el menor permite establecer diferencias entre el grupo de reincidentes y de no reincidentes. Partiendo de que las diferencias halladas son estadísticamente significativas puesto que $\times 2=19.561^{a} ; g l=3$; sig $=.000$, entre 
los reincidentes hay un porcentaje mayor de menores que han sufrido maltrato directo (3.7\%) o indirecto (6.1\%) que en el grupo de no reincidentes $(0.7 \%$ y $1.9 \%$ respectivamente).

Toda la información pormenorizada que se ha recogido sobre las personas que conviven con el menor en el momento de la causa base se ha agrupado en cuatro categorías para su análisis y comprensión: el primer grupo lo forman los niños que viven en familia de composición tradicional (padre, madre y hermanos); el segundo grupo se ha compuesto por familias constituidas solo por el padre o por la madre, el padre y los hermanos, la madre y los hermanos, o un progenitor con su pareja y hermanos; el tercer grupo está formado por la familia extensa, familia de acogida o instituciones de protección (la falta de datos nos ha impedido individualizar al grupo que procedía de instituciones de protección); y en cuarto lugar, la categoría «otros» sería el cuarto grupo, donde se ha incluido tanto a los menores que viven en la calle como, por ejemplo, los que viven con su pareja, teniendo como denominador común que en ambos casos son ellos los que tienen que responsabilizarse de su supervivencia (véase gráfico n. ${ }^{\circ} 1$ ).

\section{Gráfico 1: Convivencia del menor en la actualidad y reincidencia}

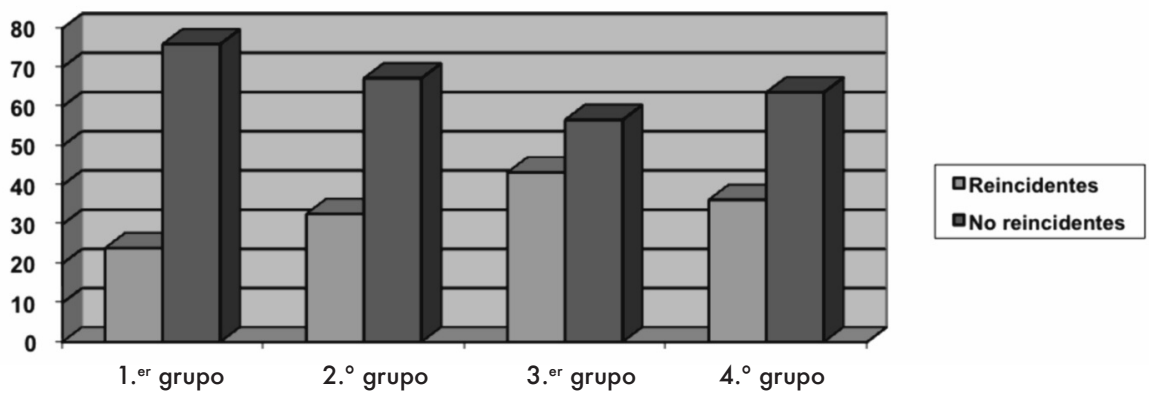

Como se muestra en el histograma, la reincidencia de los menores es cada vez mayor conforme van desapareciendo de su entorno las personas que conforman su núcleo familiar. Así, los que menos reinciden son los jóvenes del primer grupo, compuesto por los padres y hermanos; seguidos de los jóvenes del segundo grupo, que es en el que falta uno de los progenitores del menor; la tasa más alta de reincidencia la alcanza la formada por los menores del tercer grupo, que es en el que se encuentran los menores institucionalizados o con familia extensa. El cuarto grupo, que sería del que se podría esperar una mayor reincidencia por encontrarse en una situación de mayor desprotección en términos de control y afecto paterno-filial, presenta una reincidencia menor que el tercer grupo. 
De hecho, entre los menores extranjeros no acompañados $(n=10)$, la reincidencia se mantiene en un tercio, siendo las diferencias significativas $(x 2=$ $52.775^{a} ; g l=2 ; s i g=.0001$ ).

Además de la estructura familiar, nos interesa saber en qué medida los problemas que padecen las familias pueden estar relacionados con la reincidencia, partiendo de la hipótesis de que dichos problemas pueden restar atención y control al comportamiento del menor como pilares de una buena supervisión paterna (entre otros, Wasserman et al, 2003; Farrington, 2005). Las familias fueron clasificadas en función de si tenían o no problemas. El porcentaje de menores que reinciden es mayor (31.3\%) en el grupo de familias con problemas que en el de sin problemas ( $14.8 \%)$, siendo las diferencias significativas: $x 2=21.920^{a} ; g l=2 ; \operatorname{sig}=.000$.

Es frecuente relacionar los problemas económicos familiares con la delincuencia. Los resultados de nuestro estudio permiten afirmar, a partir de las diferencias estadísticamente significativas encontradas $\left(x 2=.068^{a} ; g l=1 ; \operatorname{sig}=.794\right)$, que hay más menores sin problemas económicos familiares que no reinciden (65.1\%) que los que sí lo hacen (34.9\%). No obstante, el $42 \%$ de los reincidentes tenían dificultades económicas en sus casas frente al 29.9\% de los no reincidentes.

Resultados muy parecidos a los anteriores los obtenemos al cruzar la reincidencia y la delincuencia de familiares. En el grupo de jóvenes que tienen familiares que han cometido o comenten hechos delictivos, la reincidencia es superior (43.4\%) que la no reincidencia (25.3\%). Además, entre los menores que no tienen familiares delincuentes el porcentaje de no reincidencia alcanza el $74.7 \%$, siendo las diferencias estadísticamente significativas $\left(x 2=17.714^{a}\right.$; $g l=2$; $s i g=$ .000). En este caso, las teorías del aprendizaje explicarían en mejor medida dicha hipotética relación. No obstante, se ha destacado en un estudio reciente que la conducta desviada del padre desempeña un rol más importante en la posibilidad de que un menor cometa delitos frente a la de otros miembros de la familia como la madre, abuelos, hermanos, etc. Sin embargo, no parece ser un factor que por sí solo permita explicar la delincuencia de los menores, sino que aparece conectado a otros factores. Concretamente en ese estudio, se apunta a la juventud de la madre del menor, al deterioro del barrio de residencia y al bajo sentimiento de culpabilidad (guilty) (Farrington; Jolliffe; Loeber; Stouthamer-Loeber y Kalb, 2001).

También ha sido demostrado por la literatura que el grupo de iguales está relacionado con la reincidencia. En nuestro caso, el 39.9\% de los jóvenes que frecuentaban amistades disociales reiteraron comportamientos delictivos siendo menores de edad; este porcentaje disminuye ligeramente a un 38\% para el grupo de jóvenes que solo mantenían relaciones con amigos sociales; y se re- 
duce hasta un $4.9 \%$ para los jóvenes que tienen de los dos tipos de amistades, disociales y sociales $\left(x 2=20.707^{a} ; g l=3\right.$; sig $\left.=.000\right)$.

El entorno social problemático puede entorpecer la reinserción del menor tras finalizar la medida impuesta judicialmente o incluso al tiempo de su cumplimiento. En el grupo de reincidentes, el 39.3\% vivía en un entorno de esas características. Lo más sobresaliente es que el $37.6 \%$ de los menores que tenían un entorno problemático reincidió, frente al $24.1 \%$ de los que también reincidieron pero que no se desenvolvían en un ambiente social como al que nos referimos $\left(x 2=12.220^{a} ; g l=2 ; \operatorname{sig}=.002\right)$.

Una de las formas de medir la gravedad del hecho es a partir de su consideración como delito (infracción grave) o como falta (infracción leve). Cruzando esta variable con la reincidencia, no existe una relación estadísticamente significativa entre el hecho de haber cometido una infracción calificada como delito y la repetición de infracciones posteriores. Los datos apuntan a que entre los que reincidieron, el 84\% había cometido un delito en sentido estricto que motivó la causa base. Además, el porcentaje de los que cometieron un delito y reincidieron (28.2\%) es ligeramente superior a los que cometieron una falta y reincidieron (25\%).

El $76 \%$ de los que reinciden habían cometido un delito (72.9\%) o una falta (3.1\%) contra el patrimonio (causa base). El restante $24 \%$ se distribuye entre las demás categorías delictivas. En el siguiente gráfico $n .^{\circ} 2$ puede observarse que es en los delitos contra el patrimonio donde más reincidencia posterior se produce, seguido por los delitos de atentado y resistencia a la autoridad $(x 2=$ $46.621^{a} ; g l=14 ;$ sig $=.011$.

Gráfico 2: Reincidencia según tipologías de la causa base (\%)

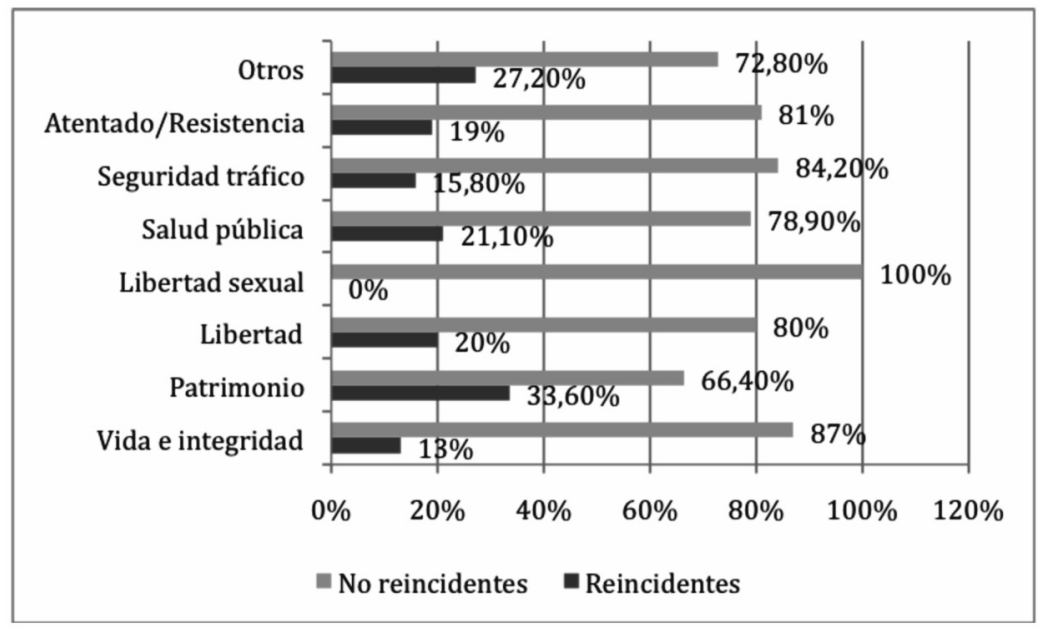


Si el grupo de iguales parece tener cierta relación con la reincidencia del menor, no se ha encontrado una relación estadísticamente significativa entre la reincidencia del sujeto y el hecho de haber actuado en la causa base en grupo, por lo que no podemos entrar a valorar la repercusión de la realización de la actividad delictiva en grupo en función a la edad y tipo de relación (mayores o menores de edad y familiares o no) de los coautores o partícipes del hecho y la reiteración de comportamientos delictivos posteriores.

Si nos centramos en las consecuencias que, en la causa base, se derivaron para el menor por su comportamiento delictivo y lo ponemos en relación con la reincidencia posterior, el resultado de dicho cruce de variables es que los menores a los que se les impuso una medida de internamiento (al $8.81 \%$ de los casos de nuestra muestra se les impuso un internamiento en régimen cerrado, semiabierto, abierto o terapéutico) reinciden con más frecuencia (55.7\% del porcentaje anterior) que los jóvenes a los que se les impuso otro tipo de medidas. Así, por ejemplo, el 36.3\% de los jóvenes a los que se les impuso una libertad vigilada reincidieron; también lo hizo el $17.4 \%$ de los que se les impuso un trabajo en beneficio de la comunidad; y el $23.2 \%$ de los que fueron amonestados. Aunque estos datos son limitados, porque difícilmente constará en los expedientes de la jurisdicción de menores la reincidencia de los menores de 16 y 17 años.

De la comparación entre reincidentes y no reincidentes según la consecuencia que se le impuso al menor en la causa base, se puede apreciar que los reincidentes destacan cuando la consecuencia impuesta es un internamiento en régimen cerrado o semiabierto. Esta relación, aunque es estadísticamente significativa $\left(x 2=47.775^{a} ; g l=13\right.$; sig = .000), no tiene una explicación simple anclada únicamente en la medida impuesta, sino que se dan otras variables, hasta el momento no aisladas en esta estudio, que pueden explicar este resultado con más profundidad. A modo de ejemplo, se advierte que no se ha ponderado la ausencia o no de discrecionalidad en la decisión judicial, tampoco los tiempos de cumplimiento de las medidas, y no se ha controlado el tiempo de exposición del menor en libertad cumpliendo o no la medida (Loughran, Mulrey, Schubert, Fagan y Piquero, 2009). Por eso hay que tomar este, y otros resultados, como meramente descriptivos y con las debidas cautelas.

También hemos cruzado el inicio o no de la medida impuesta en la causa base con la reincidencia, siendo las diferencias encontradas estadísticamente significativas $\left(x 2=4.418^{a} ; g l=1 ; s i g=.036\right)$. El resultado de tal análisis muestra que los jóvenes sobre los que se inició la medida impuesta reincidieron con mayor frecuencia (30.1\%) que aquellos menores sobre los que no se inició la medida (22.2\%). Es conocido que ni este dato ni ningún otro explican por sí 
solos la reincidencia, y que algunas variables pueden estar correlacionadas, sobreestimándose la relación entre dichas variables y la reincidencia.

Por último, parece interesante apuntar a la relación existente entre el quebrantamiento de la medida impuesta por el juez y la reiteración posterior de comportamientos delictivos. El resultado de este cruce de variables es que el $34.1 \%$ de los que quebrantaron la medida judicial de la causa base reincidieron con posterioridad. Por el contrario, de los que no quebrantaron la condena, el $27.1 \%$ no consta en su expediente judicial que hubiera reiterado un comportamiento delictivo. Aun cuando el dato descriptivo en sí nos aporta cierta información, ya que el quebrantamiento en sí debe considerarse una reincidencia en sí mismo, es importante tener en cuenta que las diferencias entre estos grupos no son estadísticamente significativas.

\section{Discusión}

Hemos aportado en esta investigación una descripción de las carreras delictivas de menores en tres provincias andaluzas a partir del análisis de los expedientes judiciales de menores. Según dichos datos, un tercio de los menores andaluces reiteran el comportamiento delictivo. Como comentábamos en la introducción, se hace difícil hacer comparaciones con otros estudios debido a la ausencia de instrumentos de medición común y a una metodología similar. Siendo este un objetivo alejado de nuestro estudio, conviene llamar la atención sobre la utilidad del uso longitudinal de este indicador para evaluar las posibles reformas legales, los cambios en los programas de tratamiento o la utilización de ciertas medidas en el ámbito de la discrecionalidad judicial.

Con independencia de lo anterior, los resultados expuestos en este trabajo nos aportan un panorama de la juventud infractora y de los elementos que permiten diferenciar a los menores reincidentes de los no reincidentes.

Según nuestros datos, el grupo de jóvenes que reincide durante su minoría de edad y, por tanto, pasa varias veces por el sistema de justicia juvenil, en contraposición a los que solo lo hacen una vez, se caracteriza por no estudiar, tener un mayor retraso escolar, un comportamiento disruptivo en el aula y problemático en general, y por tener una convivencia en mayor medida con la familia extensa, de acogida o instituciones de protección. Entre los menores reincidentes que viven con sus familias, es frecuente que estas presenten problemas no siempre relacionados con el aspecto económico y que, en porcentajes bastante elevados, tengan familiares vinculados a la delincuencia. Más de un tercio de los reincidentes tienen amistades disociales y su entorno social es problemático. El delito que más se reitera es contra la propiedad, seguido por los delitos de atentado o resistencia a la autoridad. Judicialmente, se imponen con más frecuencia medidas de internamiento a los reincidentes que a los no 
reincidentes. Y también son los reincidentes los que suelen quebrantar la medida con más frecuencia.

El análisis bivariable realizado limita la posibilidad de hacer correlaciones entre las variables estudiadas y la reincidencia de los jóvenes durante su minoría de edad. Para ello sería necesaria otra forma de medición de la reincidencia en la que se pudieran recoger los comportamientos realizados tras la mayoría de edad (especialmente para los menores que delinquen por primera vez a los 17 años) que no son detectados por el sistema de justicia juvenil. A pesar de dicha limitación, el análisis bivariable permite una aproximación descriptiva a las circunstancias que rodean a los menores infractores que pasan por el sistema de justicia juvenil y que reinciden. Variables como «familias con problemas» y «grupo de iguales disocial», entre otras, son las que en la literatura internacional (Cottle, Lee y Heilbrun, 2001) se correlacionan con la reincidencia de menores. El panorama que muestran nuestros resultados, coincidente con otros estudios, apunta a la necesidad de incidir sobre «circunstancias familiares y sociales, de personalidad e interés del menor» no solo en el ámbito del sistema de reforma, tal y como indica el artículo 7.3 de la LRRPM al señalar dichas circunstancias como criterios para adoptar la medida a imponer al menor, sino también desde el ámbito del sistema de protección de menores. Efectivamente, la LRRPM, establece que la imposición de la medida a adoptar sobre el menor infractor tiende a la prevención especial, esto es, a la no reiteración del comportamiento delictivo, actuando sobre los factores familiares, sociales y de personalidad que requieran especial atención para ese fin, siempre y cuando esas carencias no puedan ser paliadas por otras agencias de socialización informales, según el principio de subsidiariedad. Es sobradamente conocido por la literatura criminológica que el control social informal de la delincuencia, esto es, el ejercido por instancias como la familia, escuela, amigos, etc. es más eficaz contra el delito que el control social formal (policial/judicial). En la descripción bivariable expuesta de nuestra muestra se aprecia un resultado digno de resaltar y es el hecho de que los menores que han vivido con familia extensa o instituciones de protección son los que presentan una tasa de reincidencia mayor en comparación con los otros grupos. Se puede deducir de ese resultado, a modo de hipótesis, que este grupo de menores, al presentar más carencias familiares y sociales, va a tener un menor apoyo de cara a evitar una reincidencia posterior.

Nuestros resultados aportan las carencias más sobresalientes de los menores reincidentes andaluces de cara a que los operadores jurídicos y los agentes del ámbito de protección de la infancia puedan diseñar mejores estrategias de prevención secundaria y terciaria de delincuencia y persistencia del comportamiento delictivo entre este colectivo. 


\section{Referencias bibliográficas}

Aguirre Zamorano, P. (2000). Capítulo III. Las medidas. Justicia de menores: una justicia mayor. Madrid: Consejo General del Poder Judicial.

Boldova Pasamar, M. A. (2002). Principales aspectos sustantivos del nuevo Derecho penal juvenil español. Revista Aragonesa de Administración Pública, n. ${ }^{\circ}$ extra 5.

Bravo H., Sierra H. y del Valle (2007). Evaluación de resultados de medidas de responsabilidad penal juvenil en Asturias. Asturias: Dirección General de Justicia del Gobierno del Principado de Asturias.

Capdevilla, M. et al. (2005). La reincidencia en el delito en la justicia de menores. Barcelona: Centro de Estudios jurídicos y Formación especializada.

Cervelló Donderis y Colás Turégano (2002). La responsabilidad penal del menor de edad. Madrid: Tecnos.

Cottle, C. C., Lee, R. J. and Heilbrun, K. (2001). The prediction of criminal recidivism in juveniles: A meta-analysis. Criminal Justice and Behavior, 28, 3, 367-394.

Farrington, D. P. (1986). Age and Crime. Crime and Justice, 7.

Farrington, D. P., Jolliffe, D., Loeber, R., Stouthamer-Loeber, M. y Kalb, M. (2001). The concentration of offenders in families, and family criminality in the prediction of boys' delinquency. Journal of Adolescence, n. ${ }^{\circ} 24$.

Farrington, D. P. (2005). Childhood Origins Of Antisocial Behavior. Clinical Psychology and Psichotherapy, 2005, 12.

Feijoo Sánchez, B. (2001). Sobre el contenido y la evolución del Derecho penal español tras la LO 5/2000 y la LO 7/2000. Revista Jurídica de la Universidad Autónoma de Madrid, n. ${ }^{\circ} 4$.

García Pérez, O. (dir.), (2008). La delincuencia juvenil ante los Juzgados de Menores. Valencia: Tirant lo Blanch.

García Pérez, O. (2008). La reforma de 2006 del sistema español de justicia penal de menores. Política Criminal, n. ${ }^{\circ} 5$ (consultado en http://www.politicacriminal.cl/n_ 05/a_1_5.pdf).

Gómez Rivero, C. (2001). Algunos aspectos de la Ley Orgánica 5/2000, de 12 de enero, reguladora de la responsabilidad penal del menor. Actualidad Penal, n. ${ }^{\circ} 10$.

González Cussac, J. L. y Cuerda Arnau, M. L. (2002). Derecho Penal de Menores: criterios generales de aplicación de las medidas. Justicia penal de menores y jóvenes. Valencia: Tirant lo Blanch.

Gutiérrez Albentosa, J. M. (2007). Modelo de política criminal en la jurisdicción de menores. La Ley, n. ${ }^{\circ}$ 6687, de 5 de abril de 2007 (consultado en http://authn. laley.net/hdiario/2007/diario_0405_dol.html).

Gran Bretaña. Ministerio del Interior (2009). Estadísticas nacionales de Inglaterra y Gales. http://www.yjb.gov.uk/en-gb/yjs/YouthOffendingTeams/ (consultadas el 23 de octubre de 2009).

Graña et al. (2008). Infractor. Reincidencia delictiva en menores infractores en la Comunidad de Madrid: Evaluación, características delictivas y modelos de predicción. Madrid: Agencia para la Reeducación y Reinserción del Menor. 
Hagan, F. E. (2003). Research Methods in Criminal Justice and Criminology. Sexta edición. Boston: Pearson Education.

Loeber, R. y Slot, N.W. (2007). Serious and Violent Juvenile Delinquency: An Update. Crime and Justice, 35.

Loughran, Mulrey, Schubert, Fagan y Piquero (2009). Estimating a dose-response relationship between length of stay and future recidivism in serious juvenile offenders. Criminology, vol. 47, 3.

Maxfield, M. G. y Babbie, E. (2007). Basic of Research Methods for Criminal Justice and Criminology. Belnont, CA, Wadsworth Publishing. Segunda edición.

Ornosa Fernández, M. R. (2003). Derecho penal de menores. Barcelona: Bosch.

San Juan, C. y Ocaríz, E. (2009). Evaluación de la intervención y análisis de la reincidencia de la justicia de menores en la ACPV. País Vasco: Departamento de Justicia, Empleo y Seguridad Social del Gobierno Vasco.

Sánchez García de Paz (2000). La nueva Ley reguladora de la responsabilidad penal del menor. Actualidad Penal, n. ${ }^{\circ} 33$.

Tamarit Sumalla, J. M. (2001). El nuevo Derecho Penal de menores: ¿creación de un sistema penal menor?. Revista Penal, 2001, n. ${ }^{\circ} 8$.

Vargas Cabrera, B. (2001). Artículo 7. Enumeración de las medidas susceptibles de ser impuestas a los menores. La Ley de responsabilidad penal de los menores. Madrid: Trivium. 